\title{
Fixed Point Theorems and Iterative Function System in $G$-Metric Spaces
}

\author{
Salwa Salman Abed ${ }^{\mathrm{a}} \quad$ Anaam Neamah Faraj $^{\mathrm{b}}$ \\ ${ }^{a, b}$ Dep. Of Math., Coll.of Educatiuon for Pure Sciences, Ibn - Al- Haitham, Univ. of Baghdad, Iraq \\ asalwaalbundi@yahoo.com_b anaamnema1@gmail.com
}

ARTICLE INFO

Submission date: $9 / 12 / 2018$

Acceptance date: $21 / 1 / 2019$

Publication date: $16 / 2 / 2019$

\begin{abstract}
Iterated function space is a method to construct fractals and the results are self-similar. In this paper, we introduce the Hutchinson Barnsley operator (shortly, $(H-B)$ operator) on a $G$ - metric space and employ its theory to construct a fractal set as its unique fixed point by using Ciric type generalized $F$-contraction in complete $G$ - metric space. In addition, some concepts are illustrated by numerical examples.
\end{abstract}

Keywords: $G$ - metric spaces, Local fixed points, $F$ - contractions mappings. Iterated function systems.

\section{Introduction}

In mathematics there are many different definitions of fractal and have many known features, such as selfsimilarity and fractional dimension and self - similarty of iterated function system (IFS).

IFS have captivated simple spaces like square or interval [1-3]. In fact iterated function space was a method to construct fractals and the results are self-similar. Set theory are close to IFS more than fractal geometry [1].Fractals of IFS can be number of dimensions but are generally commuted and draw in 2D. The fractal is made by combination of several copies of itself, each copy transfer by a function for example canonical example for Sierpinski triangle. In case of make shapes smaller and making points close together by functions are normally contractive .

The features of (IFS) fractal was obtained by overlapping smaller copies of itself, each copy was also created by itself infinity. The process of studying fractal is an excellent science which explain possibilities in any number of application areas and in pure mathematics itself that was evolved by John Hutchinson [3] and Michael Bransley [4] and others. The iterated function system denotes theoretical straucture to follow the mathematics of many classical fractals. The attractor known as the output of an iterated function system which yeild different of fractals. The general theory of dynamical systems was embedded and appealing by mathematics of iterated function, fractals includes classical Cantor set Koch snowflake and Seirpinski gasket which generated as attraction of (IFS). Hutchinson [3] and Bransley find away to define and creat fractals as compact invariant subset of complete metric spaces with relativeto the combination of contraction Hutchinson Bransely operator (HB) known as the operator on hyoerspace of nonempty compact sets to define a fractal set as fixed point by using the theorem of Bransley in the metric spaces. By using Hutchinson Bransley test the fixed point theorem fractals [5] .Also (HB) theory developed by S.L.Singh et al. for asystem of single valued and multivalued contractions of metric spaces [6]. To prove fixed point theorem introduced K Iterated Function System using Kannan mapping in a complete metric space. Finally Easwaramoorthy et al. has investigated and generalize by (IFS) of ambiguous contractions in the fuzzy metric space [4].

In this paper, we introduce $F$ - iterated function system and employ the Hutchinson -Barnsley theory to construct a fractal set as its unique fixed point by using Ciric' type generalized F-contractions in a complete G- metric space.

"Let $\mathcal{M}$ be a nonempty set and $\Upsilon: \mathcal{M} \times \mathcal{M} \times \mathcal{M} \rightarrow \mathbb{R}^{+}$be a function satisfying the following condition :

1- $Y(q, u, v)=0$ if and only if $q=u=v$,

2- $0<Y(q, q, u), \forall q, u \in \mathcal{M}$ with $q \neq u$,

3- $Y(q, q, u) \leq Y(q, u, v)$ for all $q, u, v \in \mathcal{M}$ with $u \neq v$,

Journal of University of Babylon for Pure and Applied Sciences (JUBPAS) by University of Babylon is licened under a Creative Commons Attribution 4.0 International License. 2019. 
4- $\quad r(q, u, v)=\Upsilon(q, v, u)=\ldots$, (symmetry in all three vairables),

5- $Y(q, u, v) \leq \Upsilon(q, a, a)+\Upsilon(a, u, v)$ for all $q, u, v, a \in \mathcal{M}$.

Then the function $Y$ is called a generalized metric on $\mathcal{M}$ [7] and the pair $(\mathcal{M}, Y)$ is said a $G$-metric space." "A $G$ metric space $\mathcal{M}$ is said a symmetric [8] if $\forall r, u \in \mathcal{M}$

$$
\Upsilon(q, u, u)=\Upsilon(q, q, u) "
$$

Many results and examples about G-metric space and its generalization one can find in [9-15].

Proposition 1.1[16]: Let $(\mathcal{M}, Y)$ be a $G$-metric space, then the following are equivalent :

$1-(\mathcal{M}, Y)$ is symmetric.

$2-\gamma(q, u, u) \leq \Upsilon(q, u, a)$ for all $q, u, a \in \mathcal{M}$,

$3-\Upsilon(q, u, v) \leq \Upsilon(q, u, a)+\Upsilon(v, u, b)$ for all $q, u, v, a, b$

The $Y$-ball with center $q_{0}$ and radius $\epsilon>0$ is $B_{Y}\left(q_{0}, \epsilon\right)$ [7] is

$$
B_{\Upsilon}\left(q_{0}, \epsilon\right)=\left\{\mathrm{s} \in \mathcal{M}: \Upsilon\left(q_{0}, \mathrm{~s}, \mathrm{~s}\right)<\epsilon\right\} .
$$

The sequence $\left\{q_{n}\right\}$ in a $G$ - metric space $(\mathcal{M}, Y)$ is said to [7]

1- $\Upsilon$ - convergent to $r$ if $\exists k \in N, \epsilon>0$ for all $m, n \geq k$ such that $Y\left(q, q_{n}, q_{m}\right)<\epsilon$.

2- $\Upsilon$ - Cauchy if $\exists k \in N, \epsilon>0$ for all $m, n, l \geq k$ such that $\Upsilon\left(q_{n}, q_{m}, q_{l}\right)<\epsilon$.

A $G$-metric space $(\mathcal{M}, \Upsilon)$ is complete if every $\Upsilon$-Cauchy sequence $(\mathcal{M}, \Upsilon)$ is $\Upsilon$ - convergent in $(\mathcal{M}, \Upsilon)$.

Proposition 1.2 [17]: Let $(\mathcal{M}, Y)$ be a $G$-metric space the following statements are equivalent

$1-\left\{q_{n}\right\}$ is $Y$-convergent to $q$, if and only if $Y\left(q_{n}, q_{n}, q\right) \rightarrow 0$, as $n \rightarrow \infty$,

2- is $\Upsilon\left(q_{n} q, q\right) \rightarrow 0$ as $n \rightarrow \infty$ if and only if $\Upsilon\left(q_{n}, q_{m}, q\right) \rightarrow 0$, as $m, n \rightarrow \infty$.

Proposition 1.3 :Let $\left\{q_{n}\right\}$ and $\left\{u_{n}\right\}$ be two sequences in a $G$-metric space $(\mathcal{M}, Y)$ if $\left\{q_{n}\right\}$ converges to $q$ and $\left\{u_{n}\right\}$ converges to $u$ then $\Upsilon\left(q_{n}, q_{n}, u_{n}\right)$ converges to $\Upsilon(q, q, u)$.

The self- mapping $f$ on a G-metric space $(\mathcal{M}, \Upsilon)$ is $\Upsilon$ - continuous at $q \in \mathcal{M}$

[18] if and only if every sequence

$$
\left\{q_{n}\right\}_{n=1}^{\infty} \subset \mathcal{M} \text {, with } q_{n \rightarrow} q, \quad \text { we have } f_{q_{n}} \stackrel{\Upsilon}{\rightarrow} f_{q} "
$$

A mapping $f: \mathcal{M} \rightarrow \mathcal{M}$ be is said $F$-contraction if there exists $\tau>0$ such that $\forall q, u, v \in \mathcal{M}$,

$$
\begin{array}{r}
Y(f q, f u, f v)>0, \\
\tau+F(Y(f q, f u, f v)) \leq F(Y(q, u, v)), \quad \forall q, u, v \in \mathcal{M}
\end{array}
$$

Let $D$ be the class of all functions $F: R^{+} \rightarrow R$ is a mapping satisfying the following conditions:

(D1) $F$ is strictly increasing,

i.e. for all $q, u, v \in R^{+}$such that $q<u<v, F(q)<F(u)<F(v)$,

(D2) For each sequence $\left\{\alpha_{n}\right\}_{n=1}^{\infty}$ of positive numbers, $\lim _{n \rightarrow \infty} \alpha_{n}=0 \Leftrightarrow \lim _{n \rightarrow \infty} F\left(\alpha_{n}\right)=-\infty$.

(D3) There exist $k \in[0,1) \ni \lim _{\alpha \rightarrow 0+} \alpha^{k} F\left(\alpha_{n}\right)=0$.

Every $F$-contraction is cconstructive (from D1) and then every $F$-contraction is $Y$-continuous.

Remark 1.1 Clearly, (1.1) and (D1) implies that every $F$-contraction mapping is $\Upsilon$-continuous, since $\forall q, u, v \in \mathcal{M}$ with $f q \neq f u \neq f v$, 


$$
F(f q, f u, f v) \leq F(Y(q, u, v)) .
$$

Definition 1.1: A mapping $f$ on a $G$ - metric space $(\mathcal{M}, Y)$ is said a Ciric type generalized $F$-contraction on $(\mathcal{M}, Y)$ if $\exists$ $\tau>0$ such that for all $q, u, v \in \mathcal{M}$,

$$
[\Upsilon(f q, f u, f v)>0 \Rightarrow \tau+F(Y(f q, f u, f v)) \leq F(M(q, u, v))]
$$

where $M(q, u, v)=\max \{\Upsilon(q, u, v), \Upsilon(q, f q, f q), \Upsilon(u, f u, f u)$,

$$
\left.r(v, f v, f v), \frac{(r(q, f u, f v)+r(u, f q, f v)+r(v, f q, f u))}{3}\right\}
$$

Theorem 1.1: Let $(\mathcal{M}, Y)$ be a complete $G$ - metric space and $f: \mathcal{M} \rightarrow \mathcal{M}$ be a Ciric' type generalized $F$-contraction. If $f$ or $F$ is $Y$-continuous then $f$ has a unique fixed point in $\mathcal{M}$.

\section{Proof:}

Let $q_{0} \in \mathcal{M}$, define a sequence $\left\{q_{n}\right\}$ in $\mathcal{M}$ by $q_{n}=f q_{n-1}$ for $n \in\{1,2, \cdots\}$.

If $q_{n_{0}+1}=q_{n_{0}}$ for some $n_{0} \in\{0,1, \cdots\}$, then $f q_{n_{0}}=q_{n_{0}}$, and so, $f$ has a fixed point.

Now let $q_{n_{+1}} \neq q_{n}$ for every $n \in\{0,1, \cdots\}$ and

let

$$
y_{n}=\Upsilon\left(q_{n+1}, q_{n}, q_{n}\right), \text { for } n \in\{0,1, \cdots\}
$$

Then $y_{n}>0$ for all $n \in\{0,1, \cdots\}$.

Now using (1.2),we have

$$
\begin{aligned}
& F\left(y_{n}\right)=F\left(\Upsilon\left(q_{n+1}, q_{n}, q_{n}\right)\right) \\
& =F\left(\Upsilon\left(f q_{n}, f q_{n-1}, f q_{n-1}\right)\right) \\
& \leq F\left(\Upsilon\left(q_{n}, q_{n-1}, q_{n-1}\right)\right)-\tau \\
& =F\left(\max \left\{Y\left(q_{n}, q_{n-1}, q_{n-1}\right), Y\left(q_{n}, q_{n+1}, q_{n+1}\right)\right\}\right)-\tau \\
& =F\left(\max \left\{y_{n-1}, y_{n}, y_{n}\right\}\right)-\tau
\end{aligned}
$$

If $y_{n}>y_{n-1}$

for some $n \in\{1,2, \cdots\}$, then from (1.3) we get

$$
F\left(y_{n}\right) \geq F\left(y_{n-1}\right)-\tau,
$$

which is a contradiction, since $\tau>0$. Thus $y_{n}<y_{n-1}$ for all $n \in\{1,2, \cdots\}$

So, from (1.3) we have

$$
\begin{gathered}
F\left(y_{n}\right) \leq F\left(y_{n-1}\right)-\tau \text {. Therefore we obtain } \\
F\left(y_{n}\right) \leq F\left(y_{n-1}\right)-\tau \\
\leq F\left(y_{n-2}\right)-2 \tau \\
\cdot \\
\cdot \\
\text {. }
\end{gathered}
$$

From (1.4), we get $\lim _{n \rightarrow \infty} F\left(y_{n}\right)=-\infty$. Thus, from (D2), we have $\lim _{n \rightarrow \infty}\left(y_{n}\right)=0$.

From (D3) $\exists k \in(0,1)$ such that $\lim _{n \rightarrow \infty} y_{n}^{k} F\left(y_{n}\right)=0$.

By (1.4), the following holds for all $n \in\{1,2, \cdots\}$

$$
y_{n}^{k} F\left(y_{n}\right)-y_{n}^{k} F\left(y_{0}\right) \leq-y_{n}^{k} n \tau \leq 0 .
$$

Letting $n \rightarrow \infty$ in (1.5) we obtain that 


$$
\lim _{n \rightarrow \infty} n y_{n}^{k}=0 .
$$

From (1.6), there exits $n_{1} \in\{1,2, \cdots\}$ such that $n y_{n}^{k} \leq 1$ for all $n \geq n_{1}$.

So, we have, for all $n \geq n_{1}$

$$
y_{n} \leq \frac{1}{n^{1 / k}} .
$$

To show that $\left\{q_{n}\right\}$ is a $Y$-Cauchy sequence consider, $n \in N \ni m>n \geq n_{1}$.

From the definition of $G$ - metric and (1.7), we have

$$
\begin{aligned}
\Upsilon\left(q_{n},\right. & \left.q_{m}, q_{m}\right) \leq F\left(q_{n}, q_{n+1}, q_{n+1}\right)+F\left(q_{n+1}, q_{n+2}, q_{n+2}\right)+\cdots+F\left(q_{m-1}, q_{m}, q_{m}\right)=y_{n}+y_{n+1}+\cdots+y_{m-1} \\
& =\sum_{i=n}^{m-1} y_{i} \\
& \leq \sum_{i=n}^{\infty} y_{i} \\
& \leq \sum_{i=n}^{\infty} \frac{1}{i^{1 / k}}
\end{aligned}
$$

By the convergence of the series $\sum_{i=n}^{\infty} \frac{1}{i^{1 / k}}$, if limit $\mathrm{n} \rightarrow \infty$, we get $F\left(q_{n}, q_{m}, q_{m}\right) \rightarrow 0$.

Thus $\left\{q_{n}\right\}$ is a $Y$-Cauchy sequence in $(\mathcal{M}, Y)$. Since $\mathcal{M}$ is a complete $G$-metric space, the sequence $\left\{q_{n}\right\} \Upsilon$-converges to a point $z \in \mathcal{M}$, that is, $\lim _{n \rightarrow \infty} r_{n}=\mathrm{z}$.

First case, if $f$ is $Y$-continuous, then $z=\lim _{n \rightarrow \infty} q_{n+1}=\lim _{n \rightarrow \infty} f q_{n}=f \lim _{n \rightarrow \infty} q_{n}=f z$

and so, $z$ is a fixed point of $f$.

Second case, if $F$ is $Y$-continuous we claim that $z=f z$. Assume the contrary, that is,

$z \neq f z$.

Then $\exists n_{0} \in N$ and a subsequence $\left\{q_{n_{k}}\right\}$ of $\left\{q_{n}\right\} \ni$ for all $n_{k} \geq n_{0}, \quad Y\left(f q_{n_{k}}, f z, f z\right)>0$

(Otherwise, there exists $n_{1} \in N$ such that $q_{n}=f z$ for all $n \geq n_{1}$, which implies that $q_{n} \rightarrow f z$ it is a contradiction, since $z \neq f z$ ).

Since

$$
\Upsilon\left(f q_{n_{k}}, f z, f z\right)>0, \quad \text { for all } n_{k} \geq n_{0}
$$

then from (1.2),we have

$$
\begin{gathered}
\tau+F\left(\Upsilon\left(f q_{n_{k+1}}, f z, f z\right)\right)=\tau+F\left(Y\left(f q_{n_{k}}, f z, f z\right)\right) \leq F\left(Y\left(M\left(q_{n_{k}}, z, z\right)\right)\right. \\
\leq F\left(\max \left\{Y\left(q_{n_{k}}, z, z\right), \Upsilon\left(q_{n_{k}}, q_{n_{k+1}}, q_{n_{k+1}}\right), \Upsilon(z, f z, f z), \frac{1}{3}\left[\Upsilon\left(q_{n_{k}}, f z, f z\right)+\Upsilon(f z, f z, z)+\Upsilon\left(z, q_{n_{k+1}}, q_{n_{k+1}}\right]\right\}\right) .\right.
\end{gathered}
$$

Taking the limit $k \rightarrow \infty$ and by continuity of $F$ we have

$$
\tau+F(Y(z, f z, f z) \leq \Upsilon(z, f z, f z)),
$$

which is a contradiction. Therefore, $z=f z$.

Finally, to prove uniqueness, let $q, u \in \mathcal{M}$ and $q \neq u$ be any two fixed point of $f$, then from (1.2) we have

$$
\tau+F(r(f q, f u, f u) \leq F(M(r(q, u, u))),
$$

we obtain

$\tau+F(\Upsilon(q, u, u) \leq F(\Upsilon(q, u, u)), \quad$ which is contradiction.

Example 1.1: Let $F_{2}:(0, \infty) \rightarrow R$ be given by the formula $F_{2}(\alpha)=\alpha+\ln \alpha$. It is obvious that $F_{2} \in D$. Then each self mappings $f$ on a $G$-metric space $(\mathcal{M}, Y)$ satisfying $(1.1)$ is an $F_{2}$-contraction such that

$$
\frac{r(f q, f u, f v)}{r(q, u, v)} \mathrm{e}^{r(f q, f u, f v)-r(q, u, v)} \leq e^{-\tau},
$$

for all $q, u, v \in \mathcal{M}, \quad f q \neq f u \neq f v$ 
From the above example we conclude that every $F$-contraction $f$ is a contractive mappings, $\forall q, u, v \in \mathcal{M}, \quad f q \neq$ $f u \neq f v$.

Example 1.2 Let $\mathcal{M}=\left\{\frac{1}{n^{2}}: n \in \mathbb{N}\right\} \cup\{0\}$ and $Y(q, u, v)=|q-u|+|u-v|+|v-q|$, then $(\mathcal{M}, Y)$ is complete $G$-metric space. Define a mapping $f: \mathcal{M} \rightarrow \mathcal{M}$,

$$
f(q)= \begin{cases}\frac{1}{(n+1)^{2}}, & q=\frac{1}{n^{2}} \\ 0 & , q=0\end{cases}
$$

First, if $\quad F_{1}$ defined by $\quad F_{1}(\alpha)=\ln \alpha$. Then $f$ is not generlized $F_{1-}$ contraction that is mean $f$ is not generalized contraction of Ciric type.

We have

$$
\sup _{q, u, v \in \mathcal{M}, q \neq u \neq v} \frac{r(f q, f u, f v)}{M(q, u, v)}=1
$$

On the other hand, taking $F_{2}$ with

$$
F_{2}(\alpha)= \begin{cases}\frac{\ln \alpha}{\sqrt{\alpha}} & , 0<\alpha<e^{2} \\ \alpha-e^{2}+\frac{2}{e} & , \alpha \geq e^{2}\end{cases}
$$

In this case we find the condition $\left(D_{1}\right),\left(D_{2}\right)$ and $\left(D_{3}\right)$ satisfied for $\left(k=\frac{2}{3}\right)$ and note that $F$ is $Y$ - continuous.

To show $f$ is generalized $F_{2}$-contraction with $\tau=\ln 2$, consider

$$
\sup _{q, u, v \in \mathcal{M}} \gamma(f q, f u, f v)=1<e^{2} .
$$

$f$ is generalized $F_{2}$-contraction with $\tau=\ln 2$. If and only if , for all $q, u, v \in \mathcal{M}$

$$
\left[\Upsilon(f q, f u, f v)>0 \Rightarrow \ln 2+F_{2}(\Upsilon(f q, f u, f v)) \leq(M(q, u, v))\right]
$$

To see (1.8), it is sufficient to see that (by $\left(F_{1}\right)$ )

$\forall q, u, v \in \mathcal{M}$

$\left[\gamma(f q, f u, f v)>0 \Rightarrow \ln 2+F_{2}(\Upsilon(f q, f u, f v)) \leq F_{2}(M(q, u, v))\right]$.

$\Leftrightarrow$ For all $q, u, v \in \mathcal{M}$

$\left[\Upsilon(f q, f u, f v)>0 \Rightarrow\left[\Upsilon(f q, f u, f v)^{\frac{1}{\sqrt{Y(f q, f u, f v)}}} \Upsilon(q, u, v)^{\frac{1}{\sqrt{Y(q, u, v)}}} \leq \frac{1}{2}\right]\right.$

$\Leftrightarrow$ For all $q, u, v \in \mathcal{M}, \quad[|f q-f u|+|f u-f v|+|f q-f v|>0$

$$
\begin{aligned}
& \Rightarrow\left[(|f q-f u|+|f u-f v|+|f q-f v|)^{\frac{1}{\sqrt{|f q-f u|+|f u-f v|+|f q-f v|}}}(|q-u|+|u-v|+|q-v|)^{-\frac{1}{|q-u|+|u-v|+|v-q|}}\right. \\
& \left.\quad \leq \frac{1}{2}\right] .
\end{aligned}
$$

Now, if $q=\frac{1}{n^{2}}$ and $u=v=\frac{1}{m^{2}}$ with $u, v>q$, then

$$
\begin{aligned}
& (|f q-f u|+|f u-f v|+|f q-f v|)^{\frac{1}{\sqrt{|f q-f u|+|f u-f v|+|f q-f v|}}}(|q-u|+|u-v|+|q-v|)^{-\frac{1}{\sqrt{|q-u|+|u-v|+|q-u|}}}
\end{aligned}
$$

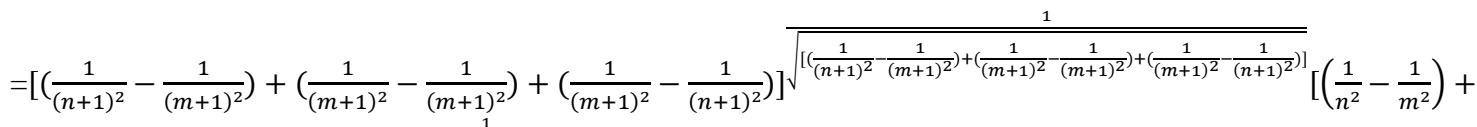

$$
\begin{aligned}
& \left.\left(\frac{1}{m^{2}}-\frac{1}{m^{2}}\right)+\left(\frac{1}{m^{2}}-\frac{1}{n^{2}}\right)\right] \sqrt{\sqrt{\left[\left(\frac{1}{n^{2}}-\frac{1}{m^{2}}\right)+\left(\frac{1}{m^{2}}-\frac{1}{m^{2}}\right)+\left[\left(\frac{1}{n^{2}}-\frac{1}{m^{2}}\right)\right.\right.}}
\end{aligned}
$$


Since $\left(\frac{m^{2}-m^{2}}{n^{2} m^{2}}\right)=0$, then

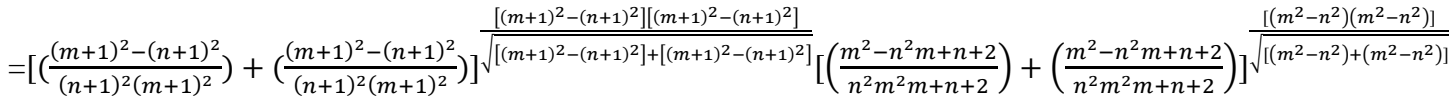

$$
\begin{aligned}
& {\left[\left(\frac{(m+1)^{2}-}{(n+1)^{2}} \frac{(n+1)^{2}}{(m+1)^{2}}\right)+\left(\frac{(m+1)^{2}-}{(m+1)^{2}} \frac{(n+1)^{2}}{(n+1)^{2}}\right)\right]^{\frac{\left[(m+1)^{2}-(n+1)^{2}\right]\left[(m+1)^{2}-(n+1)^{2}\right]}{\sqrt{\left[(m+1)^{2}-(n+1)^{2}\right]+\left[(m+1)^{2}-(n+1)^{2}\right]}}}} \\
& {\left[\begin{array}{c}
{\left[\left(\frac{(m+1)^{2}-(n+1)^{2}(m+n)(m+1)^{2}(n+1)^{2}}{(n+1)^{2}(m+1)^{2}(m+n+2) m^{2} n^{2}}\right)\right.} \\
\left.+\left(\frac{(m+1)^{2}-(n+1)^{2}(m+n)(m+1)^{2}(n+1)^{2}}{(n+1)^{2}(m+1)^{2}(m+n+2) m^{2} n^{2}}\right)\right]
\end{array}\right.} \\
& =\left[\left(\frac{(m+1)^{2}-}{(n+1)^{2}} \frac{(n+1)^{2}}{(m+1)^{2}}\right)+\left(\frac{(m+1)^{2}-}{(m+1)^{2}} \frac{(n+1)^{2}}{(n+1)^{2}}\right)\right]^{\frac{\left[(m+1)^{2}-(n+1)^{2}\right]\left[(m+1)^{2}-(n+1)^{2}\right]}{\sqrt{\left[(m+1)^{2}-(n+1)^{2}\right]+\left[(m+1)^{2}-(n+1)^{2}\right]}}} \\
& \left.\left[\left(\frac{(m+n+2) m^{2} n^{2}}{(m+n)(n+1)^{2}(m+1)^{2}}\right)+\frac{(m+n+2) m^{2} n^{2}}{(m+n)(n+1)^{2}(m+1)^{2}}\right)\right]-\frac{\left[\left(m^{2}-n^{2}\right)\left(m^{2}-n^{2}\right)\right]}{\sqrt{\left[\left(m^{2}-n^{2}\right)+\left(m^{2}-n^{2}\right)\right]}}
\end{aligned}
$$

On the other hand, since

$$
\begin{gathered}
\left(\frac{(m+1)^{2}-}{(n+1)^{2}} \frac{(n+1)^{2}}{(m+1)^{2}}\right)+\left(\frac{(m+1)^{2}-}{(m+1)^{2}} \frac{(n+1)^{2}}{(n+1)^{2}}<\frac{1}{2},\right. \\
\frac{\left[(m+1)^{2}-(n+1)^{2}\right]\left[(m+1)^{2}-(n+1)^{2}\right]}{\sqrt{\left[(m+1)^{2}-(n+1)^{2}\right]+\left[(m+1)^{2}-(n+1)^{2}\right]}} \\
-\frac{\left[\left(m^{2}-n^{2}\right)\left(m^{2}-n^{2}\right)\right]}{\sqrt{\left[\left(m^{2}-n^{2}\right)+\left(m^{2}-n^{2}\right)\right]}} \geq 1
\end{gathered}
$$

and

$$
\left(\frac{(m+n+2) m^{2} n^{2}}{(m+n)(n+1)^{2}(m+1)^{2}}\right)+\frac{(m+n+2) m^{2} n^{2}}{(m+n)(n+1)^{2}(m+1)^{2}}<1
$$

Then we get

$$
\begin{aligned}
& {\left[(|f q-f u|+|f u-f v|+|f q-f v|)^{\frac{1}{\sqrt{|f q-f u|+|f u-f v|+|f q-f v|}}}(|q-u|+|u-v|+|q-v|)\right)^{-\frac{1}{\sqrt{|q-u|+|u-v|+|q-u|}}}} \\
& \left.\leq \frac{1}{2}\right]
\end{aligned}
$$

Therefore, (1.10) is satisfied

$$
\begin{aligned}
& \text { If } q=\frac{1}{n^{2}} \text { and } u=v=0 \text {, then } \\
& \quad\left[(|f q-f u|+|f u-f v|+|f q-f v|)^{\frac{\sqrt{|f q-f u|+|f u-f v|+|f q-f v|}}{\sqrt{|c|}}}|q-u|+|u-v|+|q-v|\right)^{-\frac{1}{\sqrt{|q-u|+|u-v|+|q-u|}}} \\
& =\left|\frac{1}{(n+1)^{2}}\right| \sqrt{\frac{1}{(n+1)^{2}}}\left|\frac{1}{n^{2}}\right| \frac{1}{\sqrt{\frac{1}{n^{2}}}} \\
& =\frac{n^{2 n}}{(n+1)^{2(n+1)}}=\frac{n^{2(n+1)}}{(n+1)^{2(n+1)}} \frac{1}{n^{2}} \\
& =\left(\frac{n}{n+1}\right)^{2(n+1)} \frac{1}{n^{2}} \leq \frac{1}{2} .
\end{aligned}
$$


Then (1.9) is holds .thus all condition of theorem (1.6) are satisfied.

So, $f$ has unique fixed point in $\mathcal{M}$.

\section{2- $H B$-Operator under Ciric type generalized $F$-contraction mappings}

Definition 2.1:[19] A $\quad G-$ iterative function system (shortly, $G$ - IFS) on a $G-$ metric space $\mathcal{M}$ is a finite family of contractions $f_{n}: \mathcal{M} \rightarrow \mathcal{M}$ with contractivity factor $a_{n}$,

$n=1,2, \ldots N$. It is denoted by $\left\{\mathcal{M} ; f_{n}, n=1,2,3 \ldots \ldots N\right\}$.

Definition 2.2:[19] The general Hutchinson- Barnsley operator (shortly, HB operator) of the IFS is a function $F: 2^{(\mathcal{M})} \rightarrow 2^{(\mathcal{M})}$ defined by

$$
F(S)=\bigcup_{n=1}^{N} f_{n}(S) \text {, for all } S \in 2^{(\mathcal{M})} .
$$

Definition 2.3 [19]: The fixed point $A \in 2^{(\mathcal{M})}$ of the Hutchinson - Barnsley operator ,

$$
F(A)=\bigcup_{n=1}^{N} f_{n}(A)
$$

is called the attractor (Fractal) of the iterated function system.

Theorem 2.1: Let $(\mathcal{M}, \mathcal{Y})$ be a G-metric space and $f_{i}: \mathcal{M} \rightarrow \mathcal{M} i=\{1,2, . . k\}$ where $k$ are contraction mapping with continues $L_{1}, L_{2}, \ldots . L_{k}$. Then Hutchinson - Barnsley operator $F$ on $K(\mathcal{M})$

satisfies a contractive condition, with respect to $\Gamma$ and contractive constant $L=\max _{i=1, \ldots k} L_{i}$

i.e $\quad \Gamma(F(Q), F(U), F(V)) \leq L(Y(q, u, v)) \quad \forall q, u, v \in K(\mathcal{M})$

In particular, if $f_{i}, i=1,2, \ldots k$ are contraction mapping on $\mathcal{M}$.

Then $F$ given by (2.1), is contraction mapping of $K(\mathcal{M})$ with respect to the $\Gamma$.

Proposition 2.1: Let $(\mathcal{M}, Y)$ be a G-metric space and $\left\{f_{n}: n=1,2,3, \ldots, N\right\}$ be a sequence of a Ciric type generalized $F$-contraction on $\mathcal{M}$ with $\tau>0$ for each $f_{n}$ then $F: 2^{(\mathcal{M})} \rightarrow 2^{(\mathcal{M})}$ defined by

$$
F(S)=F_{1}(\mathrm{~S}) \cup F_{2}(\mathrm{~S}) \cup \ldots \ldots \cup F_{n}(\mathrm{~S})=\cup_{n=1}^{N} F_{n}(S), \text { for all } S \in 2^{(\mathcal{M})} .
$$

is a Ciric type generalized $F$-contraction $\left(C-F\right.$ - contraction) on $\left(2^{(\mathcal{M})}, \Gamma\right)$

Proof: Let us prove that by mathematical induction and using the properties of G-metric

For $N=1$ the statement is obviously true now let $\mathrm{N}=2$ that is

$\left[\Upsilon(f q, f u, f v)>0 \Rightarrow \tau+F\left(Y\left[\left(f_{1} \cup f_{2}\right)(q),\left(f_{1} \cup f_{2}\right)(u),\left(f_{1} \cup f_{2}\right)(v)\right]\right)\right.$

$$
\leq F(M(q, u, v),(, v, u, q)) \text {, }
$$

where

$$
\begin{gathered}
M(q, u, v)=\max \left\{\Upsilon(q, u, v), r\left(q, f_{1}(q), f_{1}(q)\right), r\left(u, f_{1}(u), f_{1}(u)\right),\right. \\
r\left(v, f_{1}(v), f_{1}(v)\right), \Upsilon\left(q, f_{2}(q), f_{2}(q)\right), r\left(u, f_{2}(u), f_{2}(u)\right), \\
r\left(v, f_{2}(v), f_{2}(v)\right), \frac{\left(r\left(q, f_{1}(u), f_{1}(v)\right)+r\left(u, f_{1}(q), f_{1}(v)\right)+\Upsilon\left(v, f_{1}(q), f_{1}(u)\right)\right)}{3} \\
\left.\frac{\left(r\left(q, f_{2}(u), f_{2}(v)\right)+r\left(u, f_{2}(q), f_{2}(v)\right)+\Upsilon\left(v, f_{2}(q), f_{2}(u)\right)\right)}{3}\right\} .
\end{gathered}
$$

Then $2 \tau+F\left\{\left[\left(\gamma\left(q, f_{1}(q), f_{1}(q)\right), \gamma\left(u, f_{1}(u), f_{1}(u)\right), \gamma\left(v, f_{1}(v), f_{1}(v)\right)\right]\right.\right.$,

$$
\left[\left(\gamma\left(q, f_{2}(q), f_{2}(q)\right), \gamma\left(u, f_{2}(u), f_{2}(u)\right), \gamma\left(v, f_{2}(v), f_{2}(v)\right)\right]\right\} \leq F(M(q, u, v),(v, u, q))
$$

$F(Y((q, q, f q)+\Upsilon(u, u, f u)+\Upsilon(v, v, f v) \leq F(M(q, u, v),(v, u, q)\}-2 \tau$.

Similarly, that is true for any natural number $\mathbb{N}$. 
Theorem 2.2: Let $(\mathcal{M}, Y)$ be complete $G$-metric space, and $\left\{\mathcal{M} ; f_{n}: n=1,2,3, \ldots, N\right\}$ a generalized $F$-contractive iteration function system. Then the following hold:

(i) A mapping $T: K(\mathcal{M}) \rightarrow K(\mathcal{M})$, where

$$
T(C)=\bigcup_{n=1}^{N} f_{n}(C) \text { for all } C \in K(\mathcal{M})
$$

is Ciric type generalized $F$-contraction on a complete $G$-metric $(K(\mathcal{M}), Y)$.

(ii) $T$ has a unique fixed point (also called an attractor or fractal).

$U \in 2^{(\mathcal{M})}, U=T(U)=\bigcup_{n=1}^{N} f_{n}(U)$.

(iii) For any initial set $C_{0} \in K(\mathcal{M})$, the sequence of compact sets $\left\{C_{0}, T\left(C_{0}\right), T^{2}\left(C_{0}\right), \ldots\right\}$ converges to a fixed point of $T$.

\section{Proof.}

Part (i) follows from proposition (2.1). For part (ii) and (iii), we proceed as follows:

Let $C_{0} \in K(\mathcal{M})$. If $C_{0}=T\left(C_{0}\right)$, then the proof is finshed.

So, assume that $C_{0} \neq T\left(C_{0}\right)$. Define

$$
C_{1}=T\left(C_{0}\right), C_{2}=T\left(C_{1}\right), \ldots, C_{m+1}=T\left(C_{m}\right), \quad \text { for } m \in N .
$$

and suppose that $C_{m} \neq C_{m+1}$, for all $m \in N$. If not , then $C_{k}=C_{k+1}$, for some $k$

implies $C_{k}=T\left(C_{k}\right)$ and this complete the proof.

Take $C_{m} \neq C_{m+1}, \quad$ for all $m \in N$. From a remark (1.4) we have

$$
\begin{gathered}
\tau\left(M_{T}\left(C_{m}, C_{m+1}, C_{m+1}\right)\right)+F\left(Y\left(C_{m+1}, C_{m+2}, C_{m+2}\right)\right)=\tau\left(M_{T}\left(C_{m}, C_{m+1}, C_{m+1}\right)\right) \\
+F\left(Y\left(T\left(C_{m}\right), T\left(C_{m+1}\right), T\left(C_{m+1}\right)\right)\right) \\
\leq F\left(M_{T}\left(C_{m}, C_{m+1}, C_{m+1}\right)\right),
\end{gathered}
$$

where

$$
\begin{aligned}
& M_{F}\left(C_{m}, C_{m+1}, C_{m+1}\right)=\max \left\{\Upsilon\left(C_{m}, C_{m+1}, C_{m+1}\right),\left(Y\left(C_{m}, T\left(C_{m}\right), T\left(C_{m}\right)\right),\left(\Upsilon\left(C_{m+1}, T\left(C_{m+1}\right), T\left(C_{m+1}\right)\right),\right.\right.\right. \\
& \frac{\left(\Upsilon\left(C_{m}, T\left(C_{m+1}\right), T\left(C_{m+1}\right)\right)+\left(\Upsilon\left(T\left(C_{m}\right),\left(C_{m+1}\right),\left(C_{m+1}\right)\right)+\left(\Upsilon\left(C_{m+1}, C_{m+1}, T\left(C_{m}\right)\right)\right.\right.\right.}{3}, \Upsilon\left(T^{2}\left(C_{m}\right), T\left(C_{m}\right), T\left(C_{m}\right)\right), Y\left(T^{2}\left(C_{m}\right), C_{m+1}, C_{m+1}\right), \\
& \left.\Upsilon\left(T^{2}\left(C_{m}\right), T\left(C_{m+1}\right), T\left(C_{m+1}\right)\right)\right\} \\
& =\max \left\{Y\left(C_{m}, C_{m+1}, C_{m+1}\right), Y\left(C_{m}, C_{m+1}, C_{m+1}\right)\left(Y\left(C_{m+1}, C_{m+2}, C_{m+2}\right)\right)\right. \text {, } \\
& \frac{\left(\left(\Upsilon\left(C_{m+1}, C_{m+2}, C_{m+2}\right)\right)+\left(\Upsilon\left(C_{m+2}, C_{m+1}, C_{m+1}\right)\right)+\left(\Upsilon\left(C_{m+1}, C_{m+1}, C_{m+1}\right)\right)\right.}{3}, \\
& \Upsilon\left(C_{m+2}, C_{m+1}, C_{m+1}\right),\left(\Upsilon\left(C_{m+2}, C_{m+1}, C_{m+1}\right)\right),\left(Y\left(C_{m+2}, C_{m+2}, C_{m+2}\right)\right) \\
& =\max \left\{\Upsilon\left(C_{m}, C_{m+1}, C_{m+1}\right),\left(\Upsilon\left(C_{m+1}, C_{m+1}, C_{m+2}\right)\right),\left(\Upsilon\left(C_{m+1}, C_{m+2}, C_{m+2}\right)\right)\right\} .
\end{aligned}
$$

In case

$M_{F}\left(C_{m}, C_{m+1}, C_{m+1}\right)=\Upsilon\left(C_{m+1}, C_{m+1}, C_{m+2}\right)$ we have

$F\left(Y\left(C_{m+1}, C_{m+1}, C_{m+2}\right) \leq F\left(Y\left(C_{m+1}, C_{m+1}, C_{m+2}\right)-\tau\left(Y\left(C_{m+1}, C_{m+1}, C_{m+2}\right)\right)\right.\right.$, a contradiction as $\tau\left(Y\left(C_{m+1}, C_{m+1}, C_{m+2}\right)\right)>0$.

Therefore, $M_{F}\left(C_{m}, C_{m+1}, C_{m+1}\right)=\Upsilon\left(C_{m+1}, C_{m+1}, C_{m+2}\right)$ and we have

$$
\begin{gathered}
F\left(Y\left(C_{m+1}, C_{m+1}, C_{m+2}\right)\right) \leq F\left(\Upsilon\left(C_{m}, C_{m+1}, C_{m+1}\right)\right)-\tau\left(\Upsilon\left(C_{m}, C_{m+1}, C_{m+1}\right)\right) \\
<F\left(\Upsilon\left(C_{m}, C_{m+1}, C_{m+1}\right)\right) .
\end{gathered}
$$

Thus $\left\{Y\left(C_{m+1}, C_{m+1}, C_{m+2}\right)\right\}$ is decreasing and hence convergent.

Now to show that

$$
\lim _{n \rightarrow \infty} \Upsilon\left(C_{m+1}, C_{m+1}, C_{m+2}\right)=0 .
$$

By property of $\tau, \exists c>0$ with $n_{0} \in N$ such that 


$$
\tau\left(\Upsilon\left(C_{m+1}, C_{m+1}, C_{m+2}\right)\right)>c \quad \forall m \geq n_{0}
$$

Note that

$$
\begin{gathered}
F\left(\Upsilon\left(C_{m+1}, C_{m+1}, C_{m+2}\right) \leq F\left(\Upsilon\left(C_{m}, C_{m+1}, C_{m+1}\right)\right)-\tau\left(\Upsilon\left(C_{m}, C_{m+1}, C_{m+1}\right)\right)\right. \\
\leq F\left(\Upsilon\left(C_{m-1}, C_{m}, C_{m}\right)\right)-\tau\left(\Upsilon\left(C_{m-1}, C_{m}, C_{m}\right)\right)-\tau\left(\Upsilon\left(C_{m}, C_{m+1}, C_{m+1}\right)\right) \\
\leq \cdots \leq\left(\Upsilon\left(C_{0}, C_{1}, C_{1}\right)\right)-\left[\tau\left(\Upsilon\left(C_{0}, C_{1}, C_{1}\right)\right)+\tau\left(\Upsilon\left(C_{1}, C_{2}, C_{2}\right)\right)\right. \\
+\cdots+\tau\left(\Upsilon\left(C_{m}, C_{m+1}, C_{m+1}\right)\right] \\
\leq F\left(\Upsilon\left(C_{0}, C_{1}, C_{1}\right)\right)-n_{0},
\end{gathered}
$$

gives $\lim _{n \rightarrow \infty} F\left(Y\left(C_{m+1}, C_{m+1}, C_{m+2}\right)=-\infty\right.$, which together with (D2) implies that

$$
\lim _{n \rightarrow \infty} \Upsilon\left(C_{m+1}, C_{m+1}, C_{m+2}\right)=0
$$

By (D3), $\exists h \in(0,1)$ such that

$$
\lim _{n \rightarrow \infty}\left[Y\left(C_{m+1}, C_{m+1}, C_{m+2}\right)\right]^{h} \cdot F\left(Y\left(C_{m+1}, C_{m+1}, C_{m+2}\right)=0 .\right.
$$

Thus we have

$$
\begin{gathered}
{\left[\Upsilon\left(C_{m}, C_{m+1}, C_{m+1}\right)\right]^{\boldsymbol{h}} F\left(\Upsilon\left(C_{m}, C_{m+1}, C_{m+1}\right)\right)-\left[\Upsilon\left(C_{m}, C_{m+1}, C_{m+1}\right)\right]^{\boldsymbol{h}} F\left(\Upsilon\left(C_{0}, C_{1}, C_{1}\right)\right)} \\
\left.\leq\left[\Upsilon\left(C_{m}, C_{m+1}, C_{m+1}\right)\right]^{\boldsymbol{h}}\left(F\left(\Upsilon\left(C_{0}, C_{1}, C_{1}\right)\right)-n_{0}\right)\right)-\left[\Upsilon\left(C_{m}, C_{m+1}, C_{m+1}\right)\right]^{\boldsymbol{h}}\left(F\left(\Upsilon\left(C_{0}, C_{1}, C_{1}\right)\right)\right. \\
\leq-n_{0}\left[\Upsilon\left(C_{m}, C_{m+1}, C_{m+1}\right)\right]^{\boldsymbol{h}} \leq 0 .
\end{gathered}
$$

On taking limit we get $\lim _{n \rightarrow \infty} m\left[\Upsilon\left(C_{m+1}, C_{m+1}, C_{m+2}\right)\right]^{h}=0$, as $n \rightarrow \infty$.

Hence

$$
\lim _{n \rightarrow \infty} m^{\frac{1}{h}} \Upsilon\left(C_{m+1}, C_{m+1}, C_{m+2}\right)=0
$$

There exists $n_{1} \in N$ such that

$m^{\frac{1}{h}} \Upsilon\left(C_{m+1}, C_{m+1}, C_{m+2}\right) \leq 1$ for all $m \geq n_{1}$ and hence

$$
\Upsilon\left(C_{m+1}, C_{m+1}, C_{m+2}\right) \leq \frac{1}{m^{1 / h}} \text { for all } m \geq n_{1} \text {. For } m, n \in N
$$

With $m>n \geq n_{1}$ we have

$$
\begin{aligned}
\Upsilon\left(C_{n}, C_{m}, C_{m}\right) \leq \Upsilon\left(C_{n}, C_{n+1}, C_{n+1}\right) & +\Upsilon\left(C_{n+1}, C_{n+2}, C_{n+2}\right)+\cdots+\Upsilon\left(C_{m-1}, C_{m}, C_{m}\right) \\
\leq & \sum_{j=n}^{\infty} \frac{1}{j^{1 / h}} .
\end{aligned}
$$

By the convergence of the series $\sum_{j=n}^{\infty} \frac{1}{j^{1 / h}}$, we get $Y\left(C_{n}, C_{m}, C_{m}\right) \rightarrow 0$ as $n, m \rightarrow \infty$.

Therefor $\left\{C_{n}\right\}$ is a $Y$ - Cuachy sequence in $\mathcal{M}$.

Since $\left(2^{(\mathcal{M})}, Y\right)$ is complete we have $C_{n} \rightarrow U$, as $n \rightarrow \infty$ for some $U \in 2^{(\mathcal{M})}$. To show $U$ is the fixed point of $T$, assume that $Y$-Hausdorff weight assign to the $U$ and $T(U)$ is not zero .

Now

$$
\begin{array}{r}
\tau\left(M_{T}\left(C_{n}, U, U\right)\right)+F\left(Y\left(C_{n+1}, T(U), T(U)\right)\right. \\
=\tau+F\left(Y\left(T\left(C_{n}\right), T(U), T(U)\right)\right) \leq F\left(\tau\left(M_{T}\left(C_{n}, U, U\right)\right),\right.
\end{array}
$$

where

$$
\begin{aligned}
M_{T}\left(C_{n}, U, U\right)= & \max \left\{\Upsilon\left(C_{n}, U, U\right), \Upsilon\left(C_{n}, T\left(C_{n}\right), T\left(C_{n}\right)\right), \Upsilon(U, T(U), T(U)),\right. \\
& \frac{\Upsilon\left(C_{n}, T(U), T(U)\right)+\Upsilon\left(T(U), T(U), C_{n}\right)+\Upsilon\left(U, T(U), T\left(C_{n}\right)\right)}{3}
\end{aligned}
$$




$$
\begin{gathered}
\left.\Upsilon\left(T^{2}\left(C_{n}\right), T\left(C_{n}\right), T\left(C_{n}\right)\right), Y\left(T^{2}\left(C_{n}\right), U, U\right), Y\left(T^{2}\left(C_{n}\right), T(U), T(U)\right)\right\} \\
=\max \left\{Y\left(C_{n}, U, U\right), Y\left(C_{n}, C_{n+1}, C_{n+1}\right), Y(U, T(U), T(U)), \frac{\gamma\left(C_{n}, T(U), T(U)\right)+\Upsilon\left(U, U, T\left(C_{n}\right)\right)+\Upsilon\left(U, U, C_{n+1}\right)}{3},\right. \\
\left.Y\left(C_{n+2}, C_{n+1}, C_{n+1}\right), Y\left(C_{n+2}, U, U\right), Y\left(C_{n+2}, T(U), T(U)\right)\right\} .
\end{gathered}
$$

Now, we consider the following cases

(i) If $M_{T}\left(C_{n}, U, U\right)=\Upsilon\left(C_{n}, U, U\right)$, then on taking lower limit as $n \rightarrow \infty$ in (2.2) we have

$$
\lim _{n \rightarrow \infty} \inf \tau\left(Y\left(C_{n}, U, U\right)\right)+F(Y(T(U), U, U) \leq F(Y(U, U, U))
$$

A contradiction as $\lim i n f_{v \rightarrow 0} \tau(v)>0$, for all $v>0$.

(i) When $M_{T}\left(C_{n}, U, U\right)=\Upsilon\left(C_{n}, C_{n+1}, C_{n+1}\right)$ then by taking lower limit as $n \rightarrow \infty$, we have

$$
\lim _{n \rightarrow \infty} \inf \tau\left(Y\left(C_{n}, C_{n+1}, C_{n+1}\right)\right)+F(Y(T(U), U, U) \leq F(Y(U, U, U)),
$$

Gives a contradiction .

(ii) In case $M_{T}\left(C_{n}, U, U\right)=\Upsilon(U, T(U), T(U))$, then we have

$$
\tau(\Upsilon(U, T(U), T(U)))+F(\Upsilon(T(U), U, U) \leq F(\Upsilon(U, T(U), T(U))),
$$

a contradiction as $\tau(Y(U, T(U), T(U)))>0$.

(iii) If $M_{T}\left(C_{n}, U, U\right)=\frac{\gamma\left(C_{n}, T(U), T(U)\right)+\Upsilon\left(U, C_{n}, C_{n}\right)+Y\left(U, C_{n+1}, C_{n+1}\right)}{3}$,

then on taking lower limit as $n \rightarrow \infty$, we get

$$
\begin{array}{r}
\lim _{n \rightarrow \infty} \inf \tau \frac{\Upsilon\left(C_{n}, T(U), T(U)\right)+\Upsilon\left(U, C_{n}, C_{n}\right)+\Upsilon\left(U, C_{n+1}, C_{n+1}\right)}{3} \\
+F\left(Y(T(U), U, U) \leq F\left(\frac{\Upsilon(U, T(U), T(U))+(\Upsilon(T(U), U, U))+(\Upsilon(U, U, U))}{3}\right)=F \frac{(\Upsilon(U, T(U), T(U))}{3},\right.
\end{array}
$$

A contradiction as $F$ is strictily increasing map.

(vii) When $M_{T}\left(C_{n}, U, U\right)=\Upsilon\left(C_{n+2}, C_{n+1}, C_{n+1}\right)$ then

$$
\lim _{n \rightarrow \infty} \inf \tau\left(Y\left(C_{n+2}, C_{n+1}, C_{n+1}\right)\right)+F(Y(T(U), U, U) \leq F(Y(U, U, U)),
$$

Gives a contradiction .

(iv) In case $M_{F}\left(C_{n}, U, U\right)=\Upsilon\left(C_{n+2}, U, U\right)$ then by taking lower limit as $n \rightarrow \infty$, we get

$$
\lim _{n \rightarrow \infty} \text { inf } \tau\left(Y\left(C_{n+2} \cdot U, U\right)\right)+F(Y(T(U), U, U) \leq F(Y(U, U, U)),
$$

Gives a contradiction

(v) Finally if $M_{T}\left(C_{n}, U, U\right)=\Upsilon\left(C_{n+2}, T(U), T(U)\right)$ then by taking lower limit as $n \rightarrow \infty$, we get

$$
\lim _{n \rightarrow \infty} \inf \tau\left(Y\left(C_{n+2} \cdot T(U), T(U)\right)\right)+F(\Upsilon(T(U), U, U) \leq F(Y(U, T(U), T(U))),
$$

a contradiction.

Hence $U$ is the fixed point of $T$.

To see $U$ is unique fixed point of $T$, assume that $U$ and $V$ are two fixed points of $T$ with $Y(U, V, V)$

is not zero.

Since $T$ is a $F$-contraction mapping we get that

$\tau\left(M_{T}(U, V, V)\right)+F(Y(U, V, V))=\tau\left(M_{T}(U, V, V)\right)+F(Y(T(U), T(V), T(V))) \leq F\left(M_{F}(U, V, V)\right)$, where

$$
\begin{gathered}
M_{T}(U, V, V)=\max \{Y(U, V, V), Y(U, T(U), T(U)), Y(V, T(V), T(V)), \\
\frac{\Upsilon(U, T(V), T(V))+Y(T(U), T(V), T(V))+\Upsilon(V, T(U), T(U))}{3}, \\
\left.Y\left(T^{2}(U), U, U\right), Y\left(T^{2}(U), V, V\right), Y\left(T^{2}(U), T(V), T(V)\right)\right\}
\end{gathered}
$$




$$
\begin{gathered}
=\max \{\Upsilon(U, V, V), \Upsilon(U, U, U), \Upsilon(V, V, V), \\
\left.\frac{\Upsilon(U, U, V)+\Upsilon(U, V, V)+\Upsilon(V, U, U)}{3}, \Upsilon(U, U, U),, \Upsilon(U, V, V),, \Upsilon(U, V, V)\right\} \\
=\Upsilon(U, V, V),
\end{gathered}
$$

That is

$$
\tau(Y(U, V, V))+F(Y(U, V, V)) \leq F(Y(U, V, V)),
$$

A contradiction as $\tau(Y(U, V, V))>0$. Thus $T$ has unique fixed point $U \in K(\mathcal{M})$.

\section{Conclusions}

This paper has been introduce $F$ - iterated function system and employ the Hutchinson -Barnsley theory to construct a fractal set as its unique fixed point by using Ciric' type generalized F-contractions in a complete G- metric space.

\section{Conflict of Interests}

There are no conflicts of interest.

\section{References}

[1] B. B. Mandelbrot, The fractal geometry of nature, vol. 173. WH freeman New York, 1983.

[2] Crownover and M. Richard, Introduction to Fractals and Chaos, Jones a Bartlett Publishers London, 1990.

[3] J. E. Hutchinson, Fractals and self similarity. University of Melbourne.[Department of Mathematics], 1979.

[4] M. F. Barnsley, Fractals everywhere. Academic press, 2014.

[5] A. Petrusel, "Fixed point theory with applications to dynamical systems and fractals" in Seminar on fixed Point Theory Cluj-Napoca, Vol. 3, pp. 305-316, 2002.

[6] S. L. Singh, B. Prasad, and A. Kumar, "Fractals via iterated functions and multifunctions," Chaos, Solitons \& Fractals, vol. 39, no. 3, pp. 1224-1231, 2009.

[7] Z. Mustafa, and B., Sims, "A New Approach to Generalized Metric Space", J. of Nonlinear and Convex Analysis Vol. 7, No. 2, pp. 289-297. 2006.

[8] S. S. Abed and H. A. Jabbar, "Coupled points for total weakly contraction mappings in $g_{m}$-spaces" International Journal of advanced Scientific Technical Research, vol. 6, No. 3, pp. 64-79, 2016.

[9] S. S. Abed and K. E. A. Sada, "Common fixed points in modular spaces," Ibn AL-Haitham J. Pure Appl. Sci., pp. 500-509, 2018.

[10] D. Wardowski, "Fixed points of a new type of contractive mappings in complete metric spaces," Fixed Point Theory Appl., Vol. 2012, No. 1, p. 94, Dec. 2012.

[11] Z. Mustafa, H. Obiedat, and F. Awawdeh, "Some fixed point theorem for mapping on complete G-metric spaces," Fixed point theory Appl., vol. 2008, no. 1, p. 189870, 2008.

[12] S. S. Abed and A. N. Faraj, "Fixed Points Results in G-Metric Spaces," Ibn AL-Haitham J. Pure Appl. Sci., vol. 32, no. 1, pp. 139-146, 2019.

[13] D. R. Sahu, A. Chakraborty, and R. P. Dubey, "K-iterated function system," Fractals, vol. 18, no. 01, pp. 139144, 2010.

[14] S. S. Abed, "Fixed Point Principles in General b-Metric Spaces and b-Menger Probabilistic spaces," J. AlQadisiyah Comput. Sci. Math., vol. 10, no. 2, p. 42, 2018.

[15] S. S. Abed and H. A. Jabbar, "Coupled points for total weakly contraction mappings via $\rho$-distance," Int. J. Basic Appl. Sci., vol. 5, no. 3, p. 164, 2016. 
[16] S. S. Abed and A. N. Faraj, "Topological properties of $G$-Hausdorff metric", International Journal of Applied Mathematics and Statistical Sciences (IJAMSS), Vol.7, No. 5, pp. 1-18, 2018.

[17] A. Kaewcharoen and A. Kaewkhao, "Common fixed points for single-valued and multi-valued mappings in Gmetric spaces,” Int. J. Math. Anal, vol. 5, no. 36, pp. 1775-1790, 2011.

[18] T. Phaneendra and K. K. Swamy, "Unique fixed point in G-metric space through greatest lower bound properties," Novi Sad J. Math, vol. 43, no. 2, pp. 107-115, 2013.

[19] R. Uthayakumar and G. A. Prabakar, "An iterated function system for Reich contraction in complete b metric space,” World Acad. Sci. Eng. Technol. Int. J. Math. Comput. Phys. Electr. Comput. Eng., vol. 7, no. 11, pp. 16401643, 2014.

\section{مبرهنات النقطة الصامدة ونظام الاوال التكرارية في فضاءات G المترية

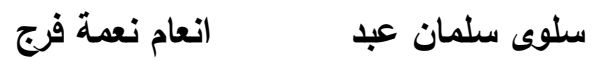 قسم الرياضيات, كلية التربية للعلوم الصرفة، /بن العيثم, جامعة بغداد} anaamnema1@gmail.com salwaalbundi@yahoo.com

في هذا البحث قدمنا مؤثر هجسن_برنسلي (بأختصار ,مؤثر H-B) على فضاءات G المترية وتوظيف الجانب النظري لأنثاء مجموعة كسورية كنقطة

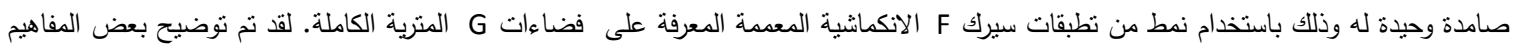
بأمثلة عددية. الكلمات الدالة: فضاءات G المترية, نقاط صامدة محلية, تطبقات F الانكماثشية, انظمة دوال تكرارية. 